\title{
Completion of Maintenance Bacillus Calmette-Guerin Therapy Might Prolong Recurrence-Free Survival in Patients with Non Muscle Invasive Bladder Cancer
}

\author{
Zaher Bahouth ${ }^{1,2 *}$, Ismail Masarwa ${ }^{1,2}$, Ofer Nativi,2, Sarel Halachmi ${ }^{1,2}$ \\ ${ }^{1}$ Department of Urology, Bnai-Zion Medical Center, Haifa, Israel \\ ${ }^{2}$ Faculty of Medicine, Technion, Haifa, Israel \\ Email: ${ }^{*}$ zaher.bahouth@b-zion.org.il
}

Received 2 May 2015; accepted 2 June 2015; published 5 June 2015

Copyright (C) 2015 by authors and Scientific Research Publishing Inc. This work is licensed under the Creative Commons Attribution International License (CC BY). http://creativecommons.org/licenses/by/4.0/

(c) $\underset{\mathrm{EY}}{\mathrm{F}}$ Open Access

\begin{abstract}
Objective: The aim of our study was to compare recurrence-free survival between patients who completed treatment with maintenance Bacillus Calmette-Guerin (BCG) and patients who did not complete the planned treatment. Materials and Methods: Data on 115 patients with intermediateand high-risk Non-Muscle Invasive Bladder Cancer (NMIBC) who were treated with BCG were available for analysis. Patients were categorized into 4 groups based on treatment duration: patients who completed three years of maintenance treatment, patients who stopped treatment while on maintenance, patients who were still on-treatment and patients who were treated with induction course only. Results: Of 115 patients, 86 were men and 29 were women with mean age of 67.8 (range 40 - 93) years. $51 \%$ had high-grade tumors and $49 \%$ had low-grade tumors. Seventythree patients $(63 \%)$ had multiple tumors. Thirty patients $(26 \%)$ were treated with inductiononly, 18 patients $(16 \%)$ are on-treatment, 14 patients $(12 \%)$ finished maintenance protocol and 53 patients $(46 \%)$ discontinued treatment. Reasons for stopping treatment were disease recurrence in 13 patients and toxicity in 40 patients. 5-year recurrence-free survival was $100 \%, 63 \%$, $60 \%$ and $56 \%$ in patients who completed maintenance treatment, stopped during maintenance treatment, were on-treatment and those who received induction only therapy, respectively. Conclusions: Patients should be encouraged to adhere to maintenance BCG treatment because of its favorable effect on recurrence-free survival probability.
\end{abstract}

\section{Keywords}

Bladder Cancer, BCG, Induction Therapy, Maintenance Treatment

\footnotetext{
${ }^{*}$ Corresponding author.
}

How to cite this paper: Bahouth, Z., Masarwa, I., Nativ, O. and Halachmi, S. (2015) Completion of Maintenance Bacillus Calmette-Guerin Therapy Might Prolong Recurrence-Free Survival in Patients with Non Muscle Invasive Bladder Cancer. Open Journal of Urology, 5, 77-83. http://dx.doi.org/10.4236/oju.2015.56012 


\section{Introduction}

Non-Muscle Invasive Bladder Cancer (NIMBC) is defined as papillary tumors confined to the mucosa or invading the lamina propria and are classified as stage Ta and T1, respectively, according to the Tumor, Node, Metastasis (TNM) classification system. Flat, high-grade tumors that are confined to the mucosa are classified as CIS (Tis). These tumors can be treated by transurethral resection of the bladder (TUR-BT) with or without intravesical instillations.

Intravesical bacillus Calmette-Guerin (BCG) is currently regarded as the standard treatment after transurethral resection for patients with NMIBC. Several randomized-controlled trials have shown the superiority of adjuvant BCG over TUR-BT alone or combined with non-BCG adjuvant intravesical therapy [1]-[3]. As BCG induces immune response, researchers developed maintenance protocols that include long-term admission of intravesical BCG beyond the induction period. Indeed, most practical guidelines recommend maintenance BCG for 1 - 3 years [4]-[6]. Several studies have shown the superiority of maintenance protocols over induction-only [2] [7]; however, recent report demonstrated no difference between the two approaches [8].

The aim of this study was to assess recurrence-free survival probabilities in patients who received complete treatment with maintenance BCG compared with those who failed to complete the full regimen.

\section{Patients and Methods}

The study was approved by the hospital IRB committee. We retrospectively evaluated data on 115 patients with NMIBC who underwent TUR-BT and were treated with intravesical BCG in our department during 1999-2010 and were followed for at least 3 years.

Most patients underwent re-TUR-BT, mainly because of pathological stage T1 or high-grade cancer. Our BCG protocol include done course (6 weekly sessions) of induction therapy followed by 7 courses ( 3 weekly sessions) of maintenance therapy over 3 years as suggested by Lamm et al. [9].

BCG treatment was started two weeks following TUR-BT. Follow-up protocol included cystoscopy, urinary tract imaging and urine cytology. The first cystoscopy was done after completing induction with 6 intravesical instillations of BCG. If no evidence of recurrence was noted, a cystoscopy was done every 3 months for the first two years, every 6 months for years 3 - 5 and annually thereafter. Urine cytology was done on each cystoscopy visit. An upper urinary tract imaging using CT-or MR-Urography was done annually.

Patients were categorized into four groups: 53 (46\%) patients who discontinued BCG while on maintenance, 18 (16\%) patients who were on-maintenance with BCG, 30 (26\%) patients who received induction therapy only and 14 (12\%) patients who completed the planned treatment protocol with BCG.

The primary endpoint was recurrence-free survival probabilities. Kaplan-Meier survival curves were constructed to estimate the recurrence-free survivalprob abilities for each group. Breslow pair wise comparison test was used to compare Kaplan-Meier curves between all groups. A sub-analysis was also done based ontumor grade. A two-sided P-value of $<0.05$ was considered as statistically significant. Statistical analyses were done using SPSS v21.

\section{Results}

Among 115 patients, 86 (77\%) were men and 29 (23\%) were women. Mean age was 67.8 years (Median 69, range 40 - 93). Multiple tumors were seen in 73 patients (63.4\%) and mean tumor size was 20.5 mm (median 19 , range 5 - $100 \mathrm{~mm}$ ). On histopathology, 59 (51\%) patients had high-grade disease and 56 (49\%) had low-grade disease. Regarding stage, 61 (53\%) patients had Ta disease, 43 (37.5\%) patients had T1 disease and 11 (9.5\%) patients had Carcinoma In Situ (CIS). Patients' baseline clinical and pathological characteristics are summarized in Table 1.

Thirty patients (26\%) received 6 instillations of induction only and discontinued treatment. Eighteen patients (16\%) are being treated with maintenance BCG, 14 patients $(12 \%)$ had completed all maintenance schedule and 53 patients $(46 \%)$ had discontinued treatment while on maintenance. Median number of maintenance courses was 3 (range 1 - 7).

Reasons for stopping treatment were recurrence in 13 patients (24.5\%) and toxicity (mainly urgency, dysuria, nocturia, fever, chills, arthritis, fatigue, abdominal pain) in 40 patients (75.5\%). Most patients had grade I adverse events as described by the NCI-CTC [10] system and 3 patients had grade II adverse events. 
With median follow-up of 77 months (IQR 58.93), disease recurrence was observed in 37 patients (32\%). Median time to recurrence was 12 months (IQR 6.30). Of these 37 patients, 14 patients (38\%) received induction therapy only, 9 patients (24\%) discontinued maintenance treatment, 13 patients (35\%) were on-maintenance treatment and only one patient (3\%) was from the completed-treatment group. Treatment and recurrence data are shown in Table 2.

Table 1. Patients’ baseline characteristics.

\begin{tabular}{cc}
\hline Age (years) & \\
Mean & 67.8 \\
Range & $40-93$ \\
Sex (\%) & $89(77 \%)$ \\
Male & $26(23 \%)$ \\
Female & \\
\hline Ethnics (\%) & $101(88 \%)$ \\
Jewish & $8(7 \%)$ \\
Arab & $6(5 \%)$ \\
Other & $59(51 \%)$ \\
Grade (\%) & $56(49 \%)$ \\
High-grade & \\
Low-grade & $61(53 \%)$ \\
\hline Stage (\%) & $43(37.5 \%)$ \\
Ta & $11(9.5 \%)$ \\
T1 & \\
CIS & $42(36.5 \%)$ \\
\hline Tumors no. (\%) & $73(63.5 \%)$ \\
Solitary & \\
Multiple & 20.5 \\
Tumor size (mm) & 19 \\
Mean &
\end{tabular}

Table 2. Treatment and recurrence data.

\begin{tabular}{cc}
\hline Treatment group (\%) & \\
Induction only & $30(26.1 \%)$ \\
Ongoing & $18(15.6 \%)$ \\
Stopped & $53(46.1 \%)$ \\
Finished & $14(12.2 \%)$ \\
\hline Maintenance cycles ${ }^{*}$ & \\
Median & 3 \\
Range & $1-7$ \\
Toxicity & $40(75.5 \%)$ \\
Recurrence & $13(24.5 \%)$ \\
\hline Recurrence rate (\%) & 37 \\
Induction only & $14(38 \%)$ \\
Ongoing & $13(35 \%)$ \\
Stopped & $9(24 \%)$ \\
Finished & $1(3 \%)$ \\
\hline Time to recurrence (mo) & 12 \\
Median & $3-66$ \\
Range & \\
\hline
\end{tabular}

* Stopped-treatment group only. 
Five-year recurrence-free survival was $100 \%, 63 \%, 60 \%$ and $56 \%$ in patients who completed the whole planned treatment, patients who discontinued treatment, patients who were on-maintenance treatment and patients who received induction only therapy, respectively. Patients who completed maintenance BCG had significantly higher recurrence-free survival rate compared to all other groups $(P=0.015)$, while no significant difference was seen between patients who discontinued treatment, patients who were on-treatment but didn't finish all courses yet and those who were treated with induction-only therapy. After stratification into high-grade and low-grade, 5-year recurrence-free survival was $87.5 \%$ and $100 \%, 71.4 \%$ and $72 \%$, 53\% and $69 \%$, 53\% and 59\% in patients who finished treatment, patients who stopped treatment, patients who were on maintenance treatment and patients who received induction-only therapy, respectively. See Figure 1, Figure 2.

\section{Discussion}

NMIBC is a chronic disease with 5-year recurrence probability of up to 78\%. Risk factors for recurrence are number of lesions, size of tumors, prior recurrence rate, stage, grade and concomitant CIS, with each additional risk factor multiplying the risk for recurrence [11]. These risk factors help stratify patients into 3 risk groups: low-risk, intermediate-risk and high-risk [4]. Patients with intermediate- and high-risk disease may have better recurrence-free survival if treated with adjuvant intravesical BCG [3] [12].

Several studies have evaluated the effect of maintenance BCG on the risk of disease progression. One large meta-analysis carried out by the EORTC-GUCG has evaluated 4863 patients in 24 different randomized control trials (RCTs). This study showed a reduction of $27 \%$ in the odds of progression with BCG maintenance treatment compared to control groups (TUR-BT alone or TUR-BT with non-BCG intravesical therapy) [7].

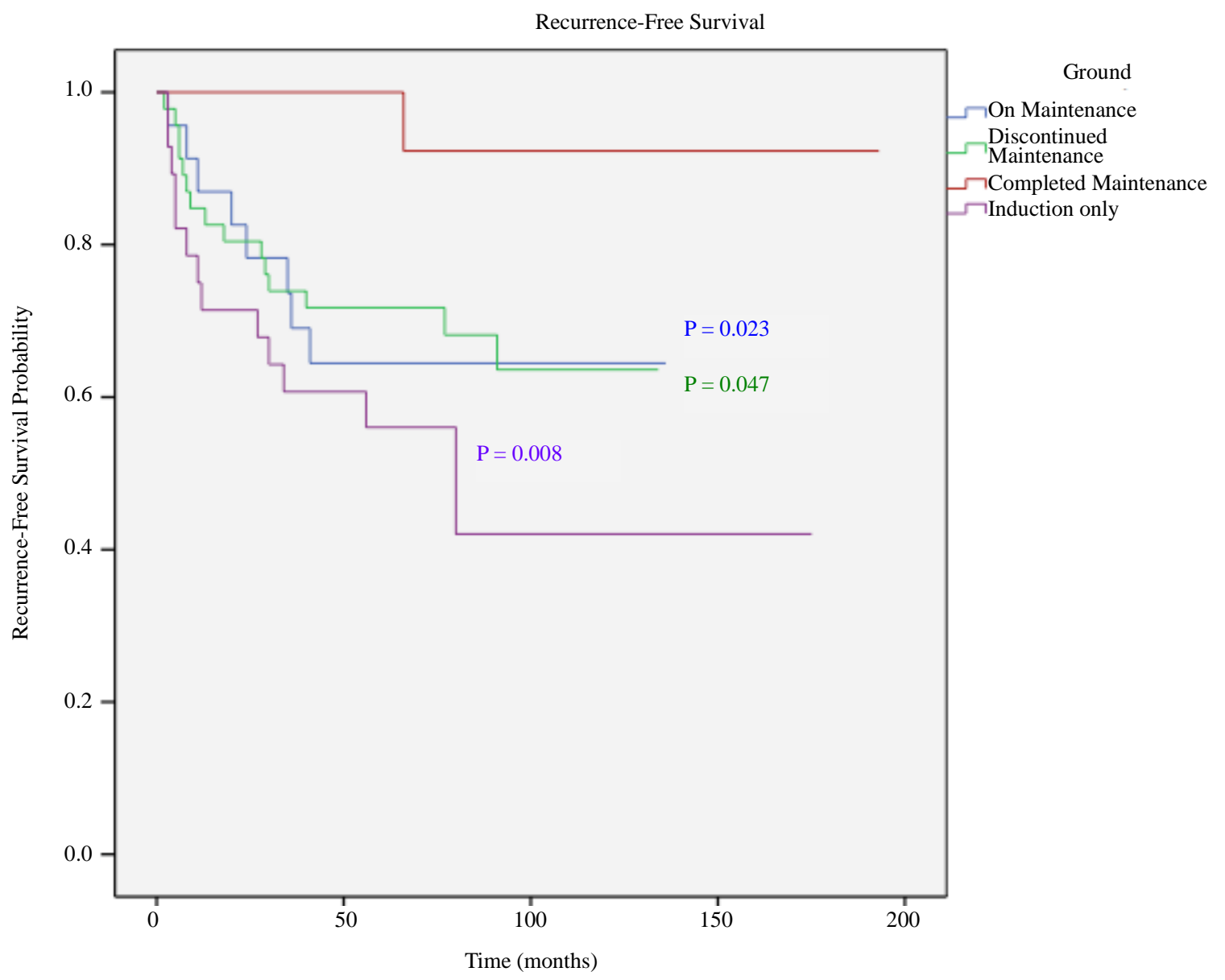

Figure 1. Overall recurrence free survival (RFS) shown as Kaplan-Meier curves in all groups. Breslow pairwise test was used to compare between KM curves. P-value indicates statistical significance compared to finished-treatment group. 

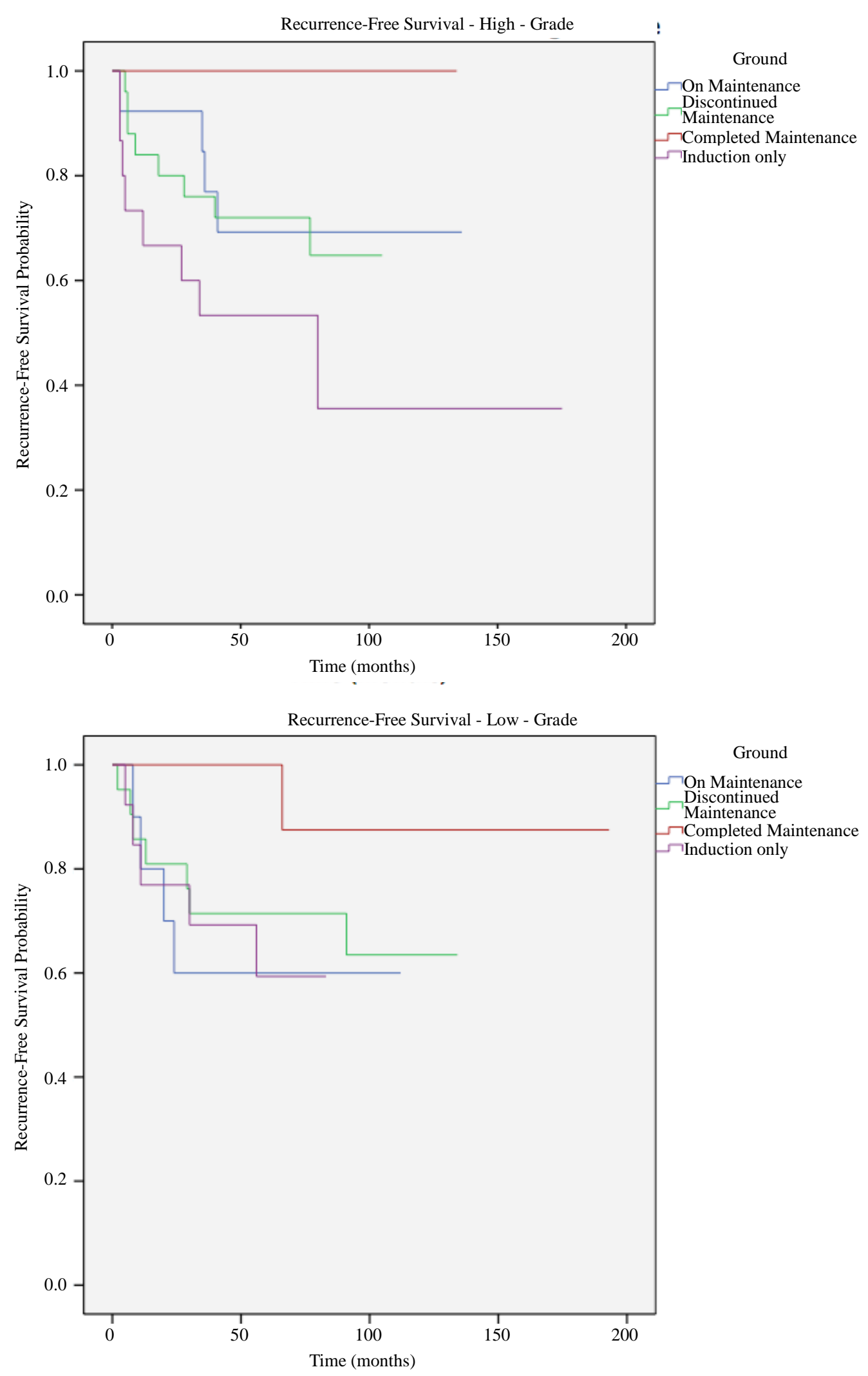

Figure 2. Recurrence-free survival adjusted to grade and shown as Kaplan-Meier curves. P-values are 0.011, $0.022,0.046$ for induction-only, ongoing and stopped-treatment groups, respectively, compared to finished-treatment group. 
On the contrary, a meta-analysis of individual patient data was not able to confirm any statistically significant difference between MMC and BCG for progression, survival and cause of death [3].

The optimal number of induction instillations and optimal frequency and duration of maintenance therapy remain unclear [13]. In a recent large RCT, the EORTC has shown that 3 years maintenance with full dose BCG reduces the recurrence rate compared to one year in high- but not in intermediate-risk patients [12].

Although concerns about BCG-associated adverse events have been expressed in the past, more recent studies have shown that maintenance schedule is not associated with an increased risk of side effects comparing to an induction course [14]. Moreover, most of the side effects can be successfully managed and those requiring treatment termination are seen more often in the first year of therapy [15].

Herr et al. questioned the need for maintenance BCG, and showed 2-year and 5-year recurrence free-survival of $73 \%$ and $46 \%$, respectively, rates that are comparable to previous studies using maintenance protocols [5]. However, most centers, including ours, still use maintenance intravesical BCG protocols for the treatment of intermediate- and high-risk NMIBC patients.

Our results showed that the effect of maintenance BCG on recurrence is in a direct duration-response relationship. Maximal long-term effect can be achieved only after several courses of BCG, ultimately after 3 years of maintenance therapy. This is demonstrated by the fact that no difference was seen between patients who didn't receive BCG compared to patients who were partially treated. We noted that patients who did not complete maintenance treatment, either because of toxicity or had not yet completed all treatment courses, had comparable recurrence-free survival rates that are significantly lower than patients who completed treatment. Conclusions cannot be made for the group of patients who was treated with induction-only because most of them stopped treatment as a result of disease recurrence.

In order to improve patients' adherence to the maintenance protocol in our center, we treat patients with oral antipyretics, analgesics and antibiotics when needed in order to reduce the irritative side effects. In some cases we reduce the dose or increase the interval between treatments sessions to help patients complete the treatment protocols.

Our study had several limitations. First of all, a small cohort that make it difficult to extrapolate the results on larger population. Another limitation is the retrospective nature and the fact that it is a non-randomized study; therefore, we cannot roll out the fact that there could be a selection bias. Further longer-term and larger studies are needed to prove, ultimately, the need for maintenance therapy, especially after several recent studies that question the need for it.

\section{Conclusion}

Our study showed that patients who completed maintenance therapy had a higher recurrence-free survival probability. Based on our results, maximal efforts should be done in order to encourage patients to adhere to the maintenance regime and to reduce the high rate of side effects related to the treatment in order to improve patients' tolerability and compliance.

\section{References}

[1] Bohle, A., Jocham, D. and Bock, P.R. (2003) Intravesical Bacillus Calmette-Guerin versus Mitomycin C for Superficial Bladder Cancer: A Formal Meta-Analysis of Comparative Studies on Recurrence and Toxicity. The Journal of Urology, 169, 90-95. http://dx.doi.org/10.1016/S0022-5347(05)64043-8

[2] Han, R.F. and Pan, J.G. (2006) Can Intravesical Bacillus Calmette-Guérin Reduce Recurrence in Patients with Superficial Bladder Cancer? A Meta-Analysis of Randomized Trials. Urology, 67, 1216-1223. http://dx.doi.org/10.1016/j.urology.2005.12.014

[3] Malmström, P.-U., Sylvester, R.J., Crawford, D.E., Friedrich, M., Krege, S., Rintala, E., et al. (2009) An Individual Patient Data Meta-Analysis of the Long-Term Outcome of Randomised Studies Comparing Intravesical Mitomycin C versus Bacillus Calmette-Guérin for Non-Muscle-Invasive Bladder Cancer. European Urology, 56, 247-256. http://dx.doi.org/10.1016/j.eururo.2009.04.038

[4] Brausi, M., Witjes, J.A., Lamm, D., Persad, R., Palou, J., Colombel, M., et al. (2011) A Review of Current Guidelines and Best Practice Recommendations for the Management of Nonmuscle Invasive Bladder Cancer by the International Bladder Cancer Group. The Journal of Urology, 186, 2158-2167. http://dx.doi.org/10.1016/j.juro.2011.07.076

[5] Lamm, D., Colombel, M., Persad, R., Soloway, M., Böhle, A., Palou, J., et al. (2008) Clinical Practice Recommendations for the Management of Non-Muscle Invasive Bladder Cancer. European Urology Supplements, 7, 651-666. 
http://dx.doi.org/10.1016/j.eursup.2008.07.009

[6] Montie, J.E., Clark, P.E., Eisenberger, M.A., El-Galley, R., Greenberg, R.E., Herr, H.W., et al. (2009) Bladder Cancer. Journal of the National Comprehensive Cancer Network, 7, 8-39.

[7] Sylvester, R.J., van der Meijden, A.P.M. and Lamm, D.L. (2002) Intravesical Bacillus Calmette-Guerin Reduces the Risk of Progression in Patients with Superficial Bladder Cancer: A Meta-Analysis of the Published Results of Randomized Clinical Trials. The Journal of Urology, 168, 1964-1970. http://dx.doi.org/10.1016/S0022-5347(05)64273-5

[8] Herr, H.W., Dalbagni, G. and Donat, S.M. (2011) Bacillus Calmette-Guérin without Maintenance Therapy for HighRisk Non-Muscle-Invasive Bladder Cancer. European Urology, 60, 32-36. http://dx.doi.org/10.1016/j.eururo.2011.03.051

[9] Lamm, D.L., Blumestein, B.A., Crissman, J.D., Montie, J.E., Gottesman, J.E., Lowe, B.A., et al. (2000) Maintenance Bacillus Calmette-Guerin Immunotherapy for Recurrent TA, T1 and Carcinoma in Situ Transitional Cell Carcinoma of the Bladder: A Randomized Southwest Oncology Group Study. The Journal of Urology, 163, 1124-1129. http://dx.doi.org/10.1016/S0022-5347(05)67707-5

[10] Trotti, A., Colevas, A.D., Setser, A., Rusch, V., Jaques, D., Budach, V., et al. (2003) CTCAE v3.0: Development of a Comprehensive Grading System for the Adverse Effects of Cancer Treatment. Seminars in Radiation Oncology, 13, 176-181. http://dx.doi.org/10.1016/S1053-4296(03)00031-6

[11] Sylvester, R.J., van der Meijden, A.P.M., Oosterlinck, W., Witjes, J.A., Bouffioux, C., Denis, L., et al. (2006) Predicting Recurrence and Progression in Individual Patients with Stage Ta T1 Bladder Cancer Using EORTC Risk Tables: A Combined Analysis of 2596 Patients from Seven EORTC Trials. European Urology, 49, 466-477. http://dx.doi.org/10.1016/j.eururo.2005.12.031

[12] Oddens, J., Brausi, M., Sylvester, R., Bono, A., van de Beek, C., van Andel, G., et al. (2013) Final Results of an EORTC-GU Cancers Group Randomized Study of Maintenance Bacillus Calmette-Guérin in Intermediate- and HighRisk Ta, T1 Papillary Carcinoma of the Urinary Bladder: One-Third Dose versus Full Dose and 1 Year versus 3 Years of Maintenance. European Urology, 63, 462-472. http://dx.doi.org/10.1016/j.eururo.2012.10.039

[13] Zlotta, A.R., van Vooren, J.P., Huygen, K., Drowart, A., Decock, M., Pirson, M., et al. (2000) What Is the Optimal Regimen for BCG Intravesical Therapy? Are Six Weekly Instillations Necessary? European Urology, 37, 470-477. http://dx.doi.org/10.1159/000020170

[14] Van der Meijden, A.P.M., Sylvester, R.J., Oosterlinck, W., Hoeltl, W. and Bono, A.V. (2003) Maintenance Bacillus Calmette-Guerin for Ta T1 Bladder Tumors Is Not Associated with Increased Toxicity: Results from a European Organisation for Research and Treatment of Cancer Genito-Urinary Group Phase III Trial. European Urology, 44, 429434. http://dx.doi.org/10.1016/S0302-2838(03)00357-9

[15] Brausi, M., Oddens, J., Sylvester, R., Bono, A., van de Beek, C., van Andel, G., et al. (2014) Side Effects of Bacillus Calmette-Guérin (BCG) in the Treatment of Intermediate- and High-risk Ta, T1 Papillary Carcinoma of the Bladder: Results of the EORTC Genito-Urinary Cancers Group Randomised Phase 3 Study Comparing One-third Dose with Full Dose and 1 Year with 3 Years of Maintenance BCG. European Urology, 65, 69-76.

http://dx.doi.org/10.1016/j.eururo.2013.07.021 